\title{
CORRIGENDUM
}

\section{Functional evaluation of pallid mice with genetic emphysema}

Mitsuteru Yoshida, Shoji Sakiyama, Koichiro Kenzaki, Hiroaki Toba, Kou Uyama, Masatsugu Takehisa, Kazuya Kondo and Akira Tangoku

Laboratory Investigation (2009) 89, 1187; doi:10.1038/labinvest.2009.72

Correction to: Laboratory Investigation (2009) 89, 760-768; advance online publication, 20 April 2009; doi:10.1038/ labinvest.2009.34

In this article, the authors have identified errors in the captions of Figures 2 and 6.

Page 763 , Figure $2 \mathrm{a}$ caption, line 3 , the second sentence should be 'The distances covered by the C57BL/6J mice at 3 months of age were significantly lower than those covered by the $\mathrm{C} 57 \mathrm{BL} / 6 \mathrm{~J}$ mice at 6 months $(P<0.01)$ and 12 months
$(P<0.01)$ of age.' Figure $2 \mathrm{~b}$, line 9 , the second sentence should be 'The TS and NS for the C57BL/6J mice at 3 months of age were significantly higher than those of the C57BL/6J mice at 6 months $(P<0.05)$ and 12 months $(P<0.05)$ of age.'

Page 767, Figure 6 caption, line 8, the second sentence should be '(c) At all ages, neither strain showed a bias of the Lm within each field (upper, middle, lower) at the same age. One-way analysis of variance did not reveal any statistically significant differences in the C57BL/6J mice and the pallid mice.' 